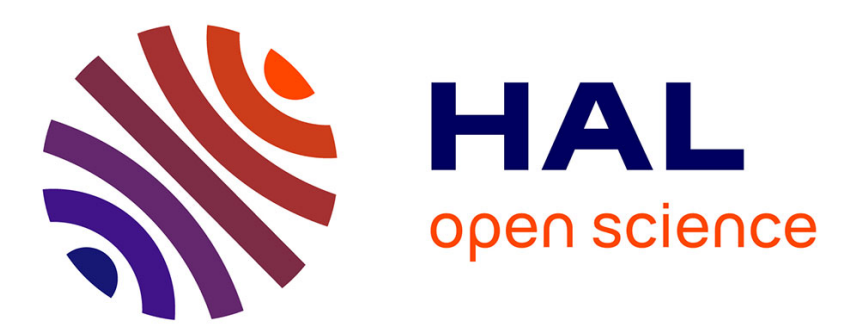

\title{
Mesurer les inégalités environnementales et écologiques dans les villes en développement : déchets et eaux usées à Lima
}

\author{
Mathieu Durand
}

\section{- To cite this version:}

Mathieu Durand. Mesurer les inégalités environnementales et écologiques dans les villes en développement : déchets et eaux usées à Lima. Flux - Cahiers scientifiques internationaux Réseaux et territoires, 2012, 89-90 (3), pp.67. 10.3917/flux.089.0067 . halshs-02195467

\section{HAL Id: halshs-02195467 \\ https://shs.hal.science/halshs-02195467}

Submitted on 26 Jul 2019

HAL is a multi-disciplinary open access archive for the deposit and dissemination of scientific research documents, whether they are published or not. The documents may come from teaching and research institutions in France or abroad, or from public or private research centers.
L'archive ouverte pluridisciplinaire HAL, est destinée au dépôt et à la diffusion de documents scientifiques de niveau recherche, publiés ou non, émanant des établissements d'enseignement et de recherche français ou étrangers, des laboratoires publics ou privés. 


\title{
Mesurer les inégalités environnementales et écologiques dans le contexte des villes en développement Les déchets et les eaux usées à Lima
}

\author{
Mathieu DURAND \\ Maître de Conférence en Aménagement de l'espace et urbanisme \\ Université du Maine (Département de Géographie) \\ Laboratoire Espaces et Sociétés (UMR ESO 6590 CNRS) \\ Mathieu.durand@univ-lemans.fr
}

\begin{abstract}
Résumé
Depuis une petite dizaine d'années, les débats sont intenses en France autour des notions d'inégalités environnementales et d'inégalités écologiques. Certaines de leurs définitions les distinguent, d'autres les confondent ou incluent l'une dans l'autre. Pour avancer dans leur compréhension, notre démarche a été de questionner les débats théoriques à partir d'un travail empirique sur la situation des déchets solides et liquides dans la ville de Lima (Pérou), en cherchant à caractériser les relations entre inégalités sociales et inégalités liées à l'environnement.

Ce travail de terrain a permis d'identifier une série d'indicateurs mesurant et appréhendant les inégalités. Ce n'est qu'une fois ce travail effectué que nous avons opéré un retour sur les différentes définitions des inégalités environnementales et écologiques. L'objectif était de mettre en adéquation les éléments compris dans les définitions avec les indicateurs de gestion des déchets à Lima. Nous avons ainsi retenu une série de définitions permettant de mettre en adéquation les deux. Ce texte cherche à retranscrire cette méthodologie de mesure, d'identification et d'analyse des inégalités environnementales et écologiques face aux déchets. Le contexte d'une ville en développement contraint à devoir s'appuyer sur des données éparses et peu nombreuses.
\end{abstract}

\footnotetext{
Abstract

Since small ten year, the debates are intense in France around the concepts of environmental inequalities and ecological inequalities. Some their definitions distinguish them, others confuse them or include one in the other. To advance in their comprehension, our step was to question the theoretical debates starting from an empirical work. They have is to leave the situation solid waste and liquids in the town of Lima, in order to seek to characterize the relations between social inequalities and inequalities related to the environment.

This work of ground made it possible to identify a series of indicators measuring and apprehending the inequalities. It is only once this carried out work which we operated a return on the various definitions of the environmental and ecological inequalities. The objective was to put in adequacy the elements included/understood in the definitions with the indicators of management of waste in Lima (Peru). We thus retained a series of definitions allowing to put in adequacy both. This text seeks to retranscribe this methodology of measurement, identification and analysis of the
} 
environmental and ecological inequalities vis-a-vis waste. Context, that of a city under development, restricted possibilities, because of the scattered character of the data available.

\section{Resumen}

Desde una década, los debates son intensos en Francia alrededor de las nociones de desigualdades ecológicas y ambientales. Algunas definiciones les distinguen, otras les confunden o incluyen una en la otra. Para avanzar en su comprensión, nuestro objetivo fue de cuestionar los debates teóricos a raíz de un trabajo empírico. Se trata apoyarse de la situación de los residuos sólidos y líquidos en la ciudad de Lima (Perú), para intentar caracterizar las relaciones entre desigualdades sociales y desigualdades vinculadas al medio ambiente.

Este trabajo de campo permitió identificar una serie de indicadores, que miden y aprehenden las desigualdades. Una vez este trabajo hecho, operamos una vuelta hacia las distintas definiciones de las desigualdades ambientales y ecológicas. La meta es adecuar los elementos incluidos en las definiciones con los indicadores de gestión de residuos en Lima. Este texto buscar transcribir esta metodología de medida, de identificación y de análisis de las desigualdades ecológicas y ambientales hacia los residuos. El contexto de una ciudad en desarrollo, restringe en el uso de datos pocos numerosos y de calidad variable.

La mobilisation des notions d'inégalités environnementales et écologiques montre aujourd'hui leur intérêt pour faire le lien entre les problématiques environnementales et sociales du développement durable. Nous ne reviendrons par sur le débat démontrant l'utilité d'allier l'observation des inégalités sociales et des inégalités relatives à l'environnement, puisque celui-ci a été amplement commenté dans l'introduction de ce numéro thématique. Nous chercherons directement à mettre en application la mesure et la compréhension des inégalités environnementales et écologiques. Ces deux concepts sont souvent mis en opposition alors que leurs conséquences affectent fréquemment les mêmes populations sur le long terme. Comme observé dans l'introduction générale de ce numéro, la définition de ces notions n'est pourtant pas encore stabilisée. Au-delà des débats théoriques, de nombreux travaux ont montré l'importance de mesurer ces inégalités à partir d'études de cas précises, afin de mettre en avant l'inadaptation des données disponibles et la nécessité de travailler sur le territoire (Theys, 2002). Ce texte ${ }^{1}$ retranscrit donc le résultat de la méthodologie utilisée pour identifier les inégalités environnementales et écologiques relatives aux déchets dans la ville de Lima.

Il ne s'agit plus ici de discuter les différentes définitions de ces notions mais, à partir de l'une d'entre elles, de tenter de mettre en place une méthodologie permettant de mesurer les inégalités sur le terrain. Il s'agit aussi de relever un défi empirique en appliquant les notions et concepts retenus à une réalité de terrain où les données ne sont pas toujours disponibles. Ces données sont d'autant plus fragiles à Lima, ville sur laquelle porte cet article, que la connaissance statistique et cartographique du territoire y est complète. Ce genre de recherche doit pouvoir permettre, a posteriori, de rediscuter les définitions proposées par les différents auteurs, sur la base d'une méthodologie opérationnelle. Ainsi, le constat scientifique des inégalités environnementales et écologiques pourra être établi. Celui-ci permettra de mieux comprendre leurs mécanismes de construction et de renforcement, puis d'entrevoir les possibilités de leur réduction. Ces inégalités sont encore plus marquées dans une ville d'Amérique Latine caractérisée par de fortes ségrégations socio-spatiales (Dubois, 2001).

${ }^{1}$ Cet article vise à détailler la méthodologie construite dans une thèse de doctorat soutenue en 2010 et intitulée « La gestion des déchets et les inégalités environnementales et écologiques à Lima, entre vulnérabilité et durabilité » (Durand, 2010). Cette thèse fut dirigée par Vincent Goueset (Université de Rennes 2) et Robert d'Ercole (Institut de Recherche pour le Développement), avec le soutien financier de l'Institut Français d'Etudes Andines. 


\section{Construire une méthodologie de recherche en s'appuyant sur la définition des concepts}

L'objectif de ce travail est de discuter les concepts suite à un travail empirique d'observation. Cette recherche de terrain doit cependant s'appuyer sur les définitions déjà existantes de ces concepts, avant d'en affiner la compréhension. Il a donc été nécessaire de les mettre en adéquation avant leur opérationnalisation sur un terrain latino-américain. Ce n'est qu'une fois la caractérisation de la gestion des déchets à Lima faite que nous avons pu opérer un retour sur les définitions des inégalités environnementales et écologiques et en dégager quelques éléments clefs.

\section{I.1.La réception et la production : deux notions clefs}

Avant de réfléchir à une définition des deux inégalités permettant de travailler sur la question des déchets, il convient de comprendre l'enjeu de la gestion de cet objet. Le terme de " déchets " est ici compris dans un sens large, incluant les déchets solides et liquides (eaux usées). Le traitement conjoint de ces deux types de déchets, généralement étudiés de façon distincte, a été privilégié car ils sont tous deux gérés selon les mêmes étapes techniques et les mêmes modalités politiques (Lebris, 2002 ; Duchemin, 2004). Par ailleurs, la question de la contamination urbaine, notamment issue de la production de déchets, prend une place de plus en plus centrale dans les préoccupations des gestionnaires de la ville et des populations.

L'observation détaillée de chacune des étapes de la gestion des déchets permet de mettre en évidence des indicateurs qui servent à caractériser les inégalités environnementales et écologiques. Ces indicateurs opérationnels, une fois caractérisés, seront mis en correspondance avec chacun des éléments des définitions théoriques des inégalités. Ces quatre étapes de gestion des déchets sont leur production, leur collecte, leur évacuation et enfin leur traitement. La ville de Lima offre la possibilité d'observer le fonctionnement spatialisé de ces quatre étapes. Il faut pour cela s'appuyer sur les données produites par les pouvoirs publics et comprendre en détail l'imbrication avec tous les réseaux informels de gestion des déchets solides et liquides. Toutes ces données sont le ciment de la réflexion autour de la construction des inégalités environnementales et écologiques.

Parmi toutes les définitions présentées dans l'introduction générale de ce numéro thématique, certaines font un usage indifférencié des inégalités écologiques et des inégalités environnementales (Laigle, 2004); d'autres questionnent l'utilisation même du mot « écologique » pour qualifier des inégalités (Bellan et al., 2007) ; d'autre encore incluent les inégalités environnementales dans les inégalités écologiques (Emelianoff, 2006). Chacun de ces auteurs a par ailleurs évolué au fur et à mesure de ses propres publications, s'appuyant sur la réflexion collective initiée au début des années 2000. Pour notre travail visant à l'élaboration méthodologique d'une application empirique, nous retiendrons les définitions proposées par Cyria Emelianoff (2010). Ce sont celles qui permettent de mieux prendre en compte ces notions par rapport aux données collectées sur le terrain. Les inégalités environnementales sont alors la différence de réception de nuisances et de risques, ainsi que la différence de réception d'aménités et de ressources. L'idée de "réception » est centrale, puisqu'elle place les individus en situation d'être impactés (positivement ou négativement) par la qualité de l'environnement urbain. Les inégalités écologiques sont au contraire définies comme la différence dans la production d'impact sur l'environnement. C'est cette fois l'idée de «production » qui est importante, car elle marque l'empreinte de la population sur son environnement. La différence entre la réception et la production d'impacts prend une place 
centrale dans notre analyse, puisqu'elle permet de mesurer les inégalités selon des indicateurs précis et selon des données préalablement existantes à Lima.

La réception de nuisances et de risques, deux des éléments de la définition des inégalités environnementales, correspond à la réception de déchets, venant contaminer les populations et leurs espaces de vie. La réception d'aménités et de ressources a un lien avec la réception de l'aménité « propreté », c'est-à-dire l'accès au service public de collecte des déchets. Au contraire, la définition des inégalités écologiques s'appuie sur la production d'impacts sur l'environnement, ici à travers la production quantitativement différenciée de déchets. Les indicateurs utilisés pour mesurer les inégalités environnementales et écologiques à Lima se fondent sur ces critères de réception et de production de déchets, ainsi que de réception du service public de propreté.

\section{I.2. La construction d'indicateur pour mesurer les inégalités}

Le croisement entre les données des étapes techniques de gestion des déchets et les éléments de définition des inégalités environnementales et écologiques est la base de la construction des indicateurs permettant de mesurer ces dernières. L'objectif est de s'appuyer sur les définitions générales données des inégalités environnementales et écologiques, puis de voire si elles sont applicables aux déchets. C'est pour cela qu'il faut mettre en adéquation, de façon pour le moment théorique les éléments de définitions avec la gestion effective des déchets. Dans la seconde partie de ce texte, cette jonction permettra de mieux caractériser les inégalités.

La spatialisation de ces indicateurs (cf. partie 2) offre une lisibilité claire de la situation. Dans un contexte où les données font quelquefois défaut, où leur qualité est parfois contestable, il nous faut identifier les indicateurs les plus simples, mais aussi les plus proches possibles de la réalité. Ils doivent donc permettre de faire le lien entre les données sociales et environnementales, ainsi qu'entre la réception et la production de biens et de maux environnementaux. La figure $n^{\circ} 1$ présente $1^{\prime}$ indicateur utilisé pour chacun des paramètres précédemment cités. Les paramètres de réception et de production s'appliquent aux aspects environnementaux des déchets. Les données sociales seront par la suite ajoutées à l'ensemble.

\begin{tabular}{|c|c|c|c|}
\hline & $\begin{array}{l}\text { Elément de } \\
\text { définition }\end{array}$ & Paramètre pris en compte & Indicateur disponible \\
\hline \multirow{3}{*}{$\begin{array}{c}\text { Inégalités } \\
\text { environnementales }\end{array}$} & $\begin{array}{l}\text { - Nuisances } \\
\text { - Risques }\end{array}$ & $\begin{array}{l}\text { Réception de } \\
\text { déchets }\end{array}$ & $\begin{array}{l}\text { Dysfonctionnement } \\
\text { du traitement des déchets }\end{array}$ \\
\hline & $\begin{array}{l}\text { - Aménités } \\
\text { - Ressources }\end{array}$ & $\begin{array}{l}\text { Réception du service } \\
\text { de propreté }\end{array}$ & $\begin{array}{l}\text { Efficacité de la collecte } \\
\text { et de l'évacuation des déchets }\end{array}$ \\
\hline & $\begin{array}{l}\text { - Inégalités } \\
\text { sociales }\end{array}$ & $\begin{array}{l}\text { Niveaux socio- } \\
\text { économiques }\end{array}$ & $\begin{array}{l}\text { Estimation des dépenses } \\
\text { par foyers }\end{array}$ \\
\hline \multirow{3}{*}{$\begin{array}{l}\text { Inégalités } \\
\text { écologiques }\end{array}$} & \multirow{2}{*}{$\begin{array}{l}\text { - Empreinte } \\
\text { écologique }\end{array}$} & Production de déchets & $\begin{array}{l}\text { Production de déchets } \\
\text { par habitant }\end{array}$ \\
\hline & & $\begin{array}{l}\text { Atténuation de l'impact } \\
\text { des déchets }\end{array}$ & $\begin{array}{l}\text { Traitement effectif des } \\
\text { déchets collectés }\end{array}$ \\
\hline & $\begin{array}{l}\text { - Inégalités } \\
\text { sociales }\end{array}$ & $\begin{array}{l}\text { Niveaux socio- } \\
\text { économiques }\end{array}$ & $\begin{array}{l}\text { Estimation des dépenses } \\
\text { par foyers }\end{array}$ \\
\hline
\end{tabular}

Figure $\mathrm{n}^{\circ} 1$. Indicateurs utilisés pour caractériser les inégalités environnementales et écologiques relatives aux déchets 
Pour mesurer de façon concrète ces éléments, nous nous sommes heurtés à la difficulté de collecter des données qualitatives précises, fiables, et homogènes sur l'ensemble de la ville. Les nuisances sont parfois mesurées (présence de plomb dans le sang lié à la crémation de déchets, maladies de peau, etc.), cependant le lien entre déchets et contamination n'est pas directement faisable et mériterait une étude épidémiologique poussée et localisée. Ceci n'est pas notre objectif. La solution adoptée pour mesurer les sources de nuisances et les localiser spatialement a alors été de s'appuyer sur le discours des différents acteurs de terrain (pouvoirs publics, riverains, travailleurs, etc.) et de comprendre les dysfonctionnements dans les services de gestion. Nous partons ainsi du postulat que la seule présence de déchets est source de risques. Une série d'éléments géo-localisables a ainsi été identifiée.

\section{I.3.Les indicateurs environnementaux}

Les indicateurs environnementaux concernent trois éléments compris dans les définitions des inégalités environnementales et écologiques : les nuisances et les risques d'une part, les aménités et les ressources d'autre part et enfin l'empreinte écologique.

- Les nuisances et les risques consécutifs à la gestion des déchets se mesurent à Lima grâce à la réception de déchets. Celle-ci a lieu à deux échelles. Il s'agit tout d'abord de la non évacuation des déchets dans les quartiers populaires qui revient pour un foyer à réceptionner (ou plutôt à devoir conserver) ses propres déchets et à être affecté par leur présence, la capacité à faire évacuer les déchets (par un service public ou par réseaux informels) permettant au contraire de limiter les risques. La seconde échelle est celle de la ville, puisque les déchets collectés sont simplement déplacés depuis leurs lieux de production vers d'autres territoires spécifiques. Si une part importante des déchets solides $(86 \%)$ est alors traitée correctement, seules $14 \%$ des eaux usées le sont ${ }^{2}$, aboutissant à des risques très importants pour les populations habitant les espaces de réception. Pour mesurer les nuisances, il faut donc identifier les lieux recevant les déchets collectés dans l'ensemble de la ville.

- Les aménités et les ressources se mesurent grâce à la réception de l'aménité « propreté », c'est-à-dire à l'accès au service public de collecte des déchets. Environ $87 \%$ des eaux usées produites par les liméniens sont ainsi collectées, pour un chiffre équivalent concernant les déchets solides. Ces aménités seront mesurées précisément par les taux d'accès aux réseaux de collecte et par la bonne évacuation des déchets dans les différents quartiers.

- L'empreinte écologique est enfin mesurée par « la surface correspondante de terre [...] nécessaire pour la production des ressources utilisées et l'assimilation des déchets produits par une population » (Wackernagel \& Rees, 1999). Nous considérerons que plus la quantité de déchets produits par habitant est importante, plus l'empreinte écologique d'une population est élevée. Il faut ensuite relativiser cette observation par rapport à l'efficacité du traitement des déchets. L'empreinte sera ainsi bien plus grande pour les eaux usées de Lima (très peu traitées) que pour ses déchets solides (faisant l'objet d'une élimination qui s'améliore très rapidement depuis l'an 2000).

\footnotetext{
${ }^{2}$ L'ensemble des données chiffrées présentées dans ce texte a été collecté entre 2006 et 2010 à Lima. Les données relatives aux déchets solides proviennent pour l'essentiel des municipalités provinciales de Lima et de Callao. Les données relatives aux usées ont été produite par l'entreprise d'eau de Lima (SEDAPAL - Servicio de Agua Potable de Lima) et par l'Institut National de Statistique (INEI - Instituto Nacional de Estadísticas e Informática).
} 


\section{I.4. Les indicateurs sociaux}

En parallèle des données environnementales, nous avons besoin de données sociospatiales pour caractériser les inégalités. Or, comme dans la plupart des villes d'Amérique Latine (Goueset, 2006), elles ne sont pas de bonne qualité à Lima. Il est par exemple impossible de mesurer les niveaux de revenu des populations, puisque l'informalité représente $60 \%$ des activités économiques du Pérou (Barrangán, 2005). Les données environnementales montrent une pollution très localisée, ce qui nous impose d'obtenir des données sociales à une échelle très fine. Or l'essentiel des données disponibles à Lima le sont à l'échelle des districts, c'est-à-dire à l'échelle des 49 subdivisions administratives de la ville regroupant de 200 habitants à 900000 habitants. Cette échelle permet d'identifier certaines inégalités, mais n'offre pas un panorama précis des réalités.

Il faut pour identifier clairement les inégalités environnementales et écologiques, descendre à l'échelle des îlots. Les nombreux travaux de sociologues (Calderon, 2009, Riofrio, 2009) sur les évolutions récentes de la ville permettent de comprendre la construction de certaines inégalités sociales, mais n'offrent pas de panorama statistique et cartographié des inégalités sociales. Ces études montrent par ailleurs que la ségrégation s'atténue à l'échelle métropolitaine mais s'accentue aux échelles fines, que les inégalités sociales tendent à être de plus en plus reléguées dans les nouvelles périphéries ou dans des quartiers péricentraux très localisés. Dans les deux cas, il ne s'agit plus d'immenses quartiers très peuplés, mais de petits groupes de maisons en marge des anciens «bidonvilles ». La dispersion et la petite taille de ces nouveaux quartiers les rend plus difficilement identifiables.

Ce paramètre donne de l'importance au travail à une échelle fine, surtout que celle-ci est également très importante dans la gestion des déchets. Pour cela, nous nous appuierons donc sur des données provenant du recensement du logement de 2007 (INEI). Une collaboration avec les économistes de l'IEP ${ }^{3}$ (Instituto de Estudios Peruanos) a permis de calculer une estimation des dépenses moyennes des ménages à l'échelle des îlots. Ce travail, retranscrit dans la figure $n^{\circ} 6$ faisant la synthèse de l'ensemble des résultats de cette recherche, permet de spatialiser les ségrégations socio-résidentielles à Lima. La figure $\mathrm{n}^{\circ} 2$ permet pour le moment de spatialiser l'évolution historique et la ségrégation socio-spatiale à Lima. Les premiers quartiers des centres historiques de Lima et Callao sont aujourd'hui socialement mixtes. Le secteur dit de la "ville moderne » correspond à la partie située au sud du centre de Lima. Il s'agit de quartiers regroupant l'essentiel des populations aisées et des activités économiques. Enfin, les trois périphéries populaires (nord, sud et est) sont de construction récente (postérieure à 1957) et concentrent l'essentiel des populations pauvres.

${ }^{3}$ Cette méthodologie n'est que l'une de celles permettant de cartographier les ségrégations sociales à l'échelle de la ville. Comme toutes les autres, elle a certaines limites et imprécisions. Elle offre cependant un panorama le plus précis possible, dans le contexte d'une ville en développement. Nous remercions Oscar Madalengoitia et Raphael Saldaña (IEP) pour leur appui sur ce point. 


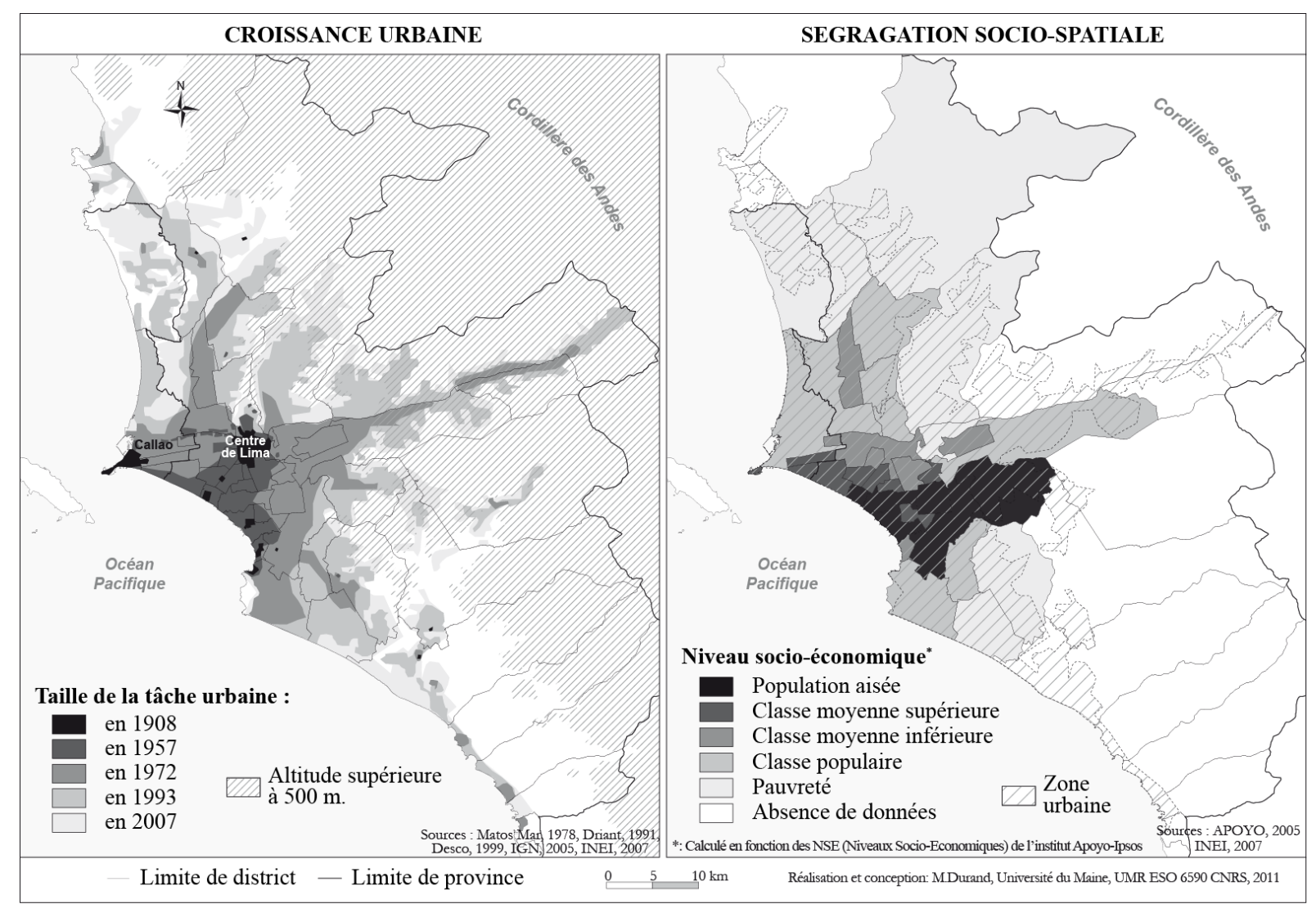

Figure $\mathrm{n}^{\circ} 2$. Une croissance urbaine marquée par la ségrégation socio-spatiale à Lima

\section{II.La représentation spatiale des inégalités environnementales et écologiques relatives aux déchets à Lima}

La méthodologie synthétisée précédemment permet d'aboutir à une cartographie précise des inégalités environnementales et écologiques par rapport aux déchets de Lima. Les cartes ci-dessous sont la synthèse d'un grand nombre de données qui ne sont pas détaillées ici, pour une question de lisibilité des résultats (Durand, 2010). Chaque donnée, correspond à une des trois parties de ce chapitre, explicitant un des éléments de la définition des inégalités environnementales et écologiques. L'ordre utilisé ici est celui des étapes techniques de la gestion des déchets (la production, la collecte, puis le traitement) et non pas l'ordre des éléments de définition utilisé précédemment dans la figure $\mathrm{n}^{\circ} 1$. La troisième partie permettra cependant de retrouver l'adéquation entre étapes techniques éléments de définition.

Les données mobilisées sont relatives au traitement des déchets solides et des eaux usées à Lima. La gestion des déchets solides est, pour ce qui concerne l'étape de la collecte, la responsabilité des 49 municipalités de districts de l'agglomération urbaine (cf. figure $\mathrm{n}^{\circ} 2$ ). L'étape du traitement est quant à lui de la responsabilité des deux municipalités de provinces, échelon administratif supérieur. On observe donc une grande fragmentation spatiale de cette gestion, sans aucun mécanisme de coordination ${ }^{4}$.

Les eaux usées sont quant à elles gérées, tout comme l'eau potable, par une entreprise dépendant directement de l'Etat péruvien, la SEDAPAL (Servicio de Agua Potable de Lima). Les pouvoirs publics locaux ne sont pas du tout représentés dans cette entreprise. Elle a, pour la partie assainissement, pendant longtemps privilégié l'accès au service de collecte au

${ }^{4}$ Il n'y a aucun lien entre les deux échelons de municipalités. Les provinces ne peuvent que très peu imposer leurs décisions aux districts. 
détriment du traitement des eaux usées, moins visible politiquement. L'articulation des activités de SEDAPAL avec les politiques municipales est donc souvent faible.

\section{II.1. Une production de déchets quantitativement différenciée}

La première étape de la mise en évidence des inégalités environnementales et écologiques est l'identification des volumes de déchets produits par les habitants des différents districts de Lima. Certaines populations ont une production de déchets plus importante que d'autres. Il s'agit de différencier spatialement les quartiers où vivent les populations ayant une empreinte écologique potentiellement forte, du fait de leur production élevée de déchets. Le terme « potentiel » fait ici référence au fait que la production de déchets sera ensuite relativisée en fonction de l'effectivité de l'élimination des déchets. La production de déchets solides provient de données de production moyenne de déchets municipaux, issues des estimations réalisées par les municipalités provinciales de Lima et de Callao ${ }^{5}$. Celle des eaux usées correspond à une estimation faite en fonction de la consommation d'eau potable par habitant.

Dans les deux cas, la production élevée de déchets est aussi bien le fruit des différences de production de déchets domestiques par individus, que de la forte concentration d'activités commerciales dans certains quartiers. Les districts des périphéries populaires Nord, Est et Sud apparaissent comme étant ceux où la population produit la moins grande quantité de déchets, malgré une certaine activité commerciale. Les districts centraux, les plus aisés, sont ceux qui produisent le plus de déchets. Les districts balnéaires du nord et du sud produisent également beaucoup de déchets à cause de l'afflux saisonnier de touristes. On note une petite différence dans certaines périphéries entre déchets solides et liquides, du fait de l'importance des réseaux techniques structurels pour les seconds.

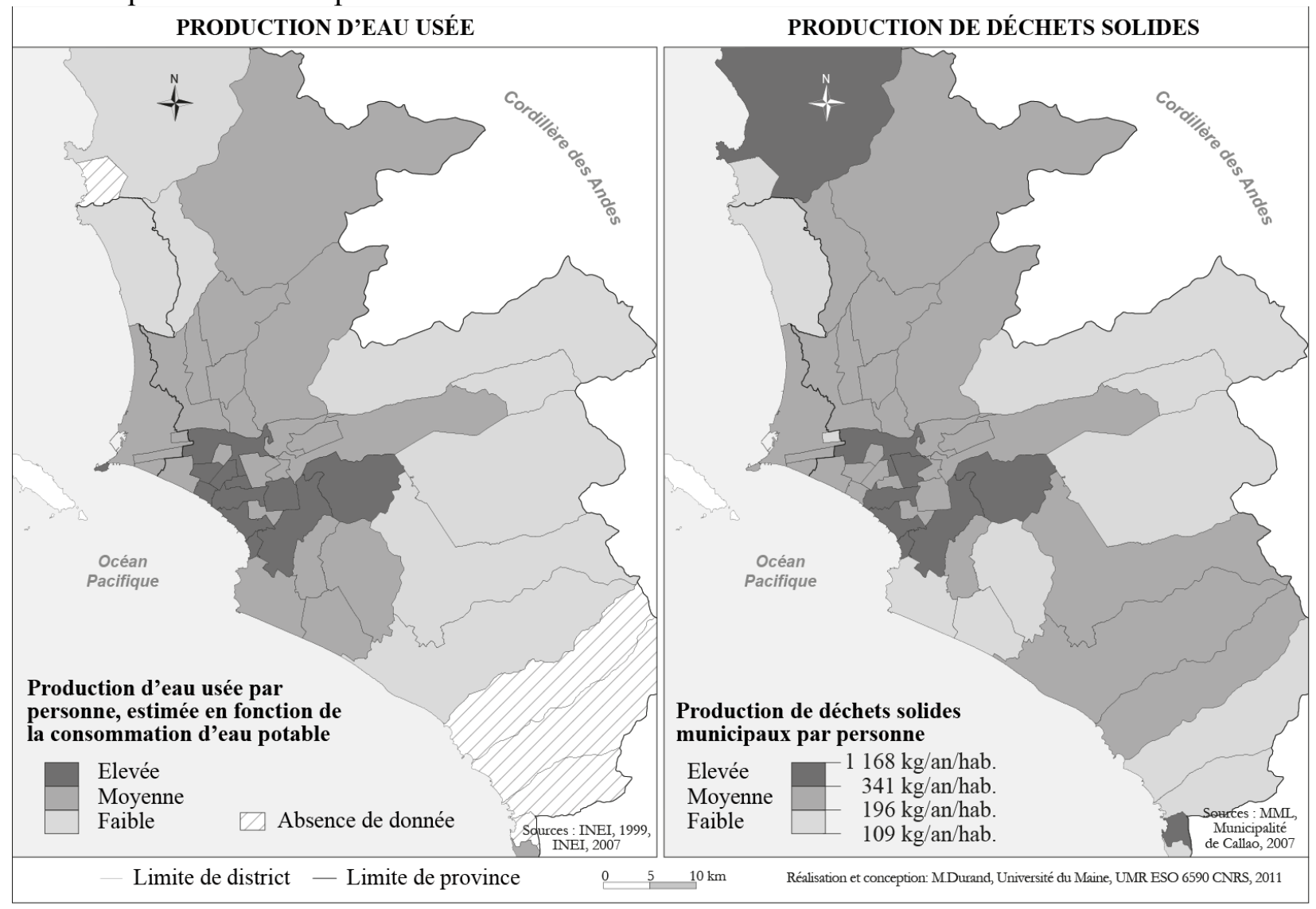

\footnotetext{
${ }^{5}$ Callao constitue la zone du port de l'agglomération de Lima
} 
Figure $\mathrm{n}^{\circ}$ 3. Synthèse de la production de déchets par habitant dans les districts de Lima en 2007

\section{II.2. L'accès au service public de propreté : la réception d'une aménité}

La seconde étape de l'identification des inégalités environnementales et écologiques se fait à travers la synthèse de l'accès au service public de collecte des déchets. Celui-ci est considéré comme une aménité puisqu'il permet d'évacuer la nuisance de son lieu de production. Nous prenons alors en compte les étapes techniques de la collecte et du transport des déchets. Les données sont légèrement différentes pour les deux catégories de déchets. L'indicateur pris en compte pour les déchets solides est celui du taux de contrôle qui correspond à la part des déchets entrant dans les cinq décharges contrôlées de la ville, par rapport à la quantité totale de déchets solides produits, selon les estimations des deux municipalités de province de Lima et de Callao. Ces informations sont disponibles à l'échelle des districts. Il s'agit donc, non pas des déchets réellement collectés, mais d'une estimation de la proportion de déchets mis en décharge. Concernant les eaux usées, le chiffre correspond à la proportion exacte de foyers ayant accès au service de collecte. Ces données sont tirées du recensement de l'INEI de 2007. L'autre distinction majeure vient du fait que les taux d'accès au réseau de collecte des eaux usées sont stables dans le temps, puisqu'ils dépendent d'infrastructures lourdes. Le niveau de contrôle des déchets solides évolue au contraire parfois rapidement en fonction des politiques municipales.

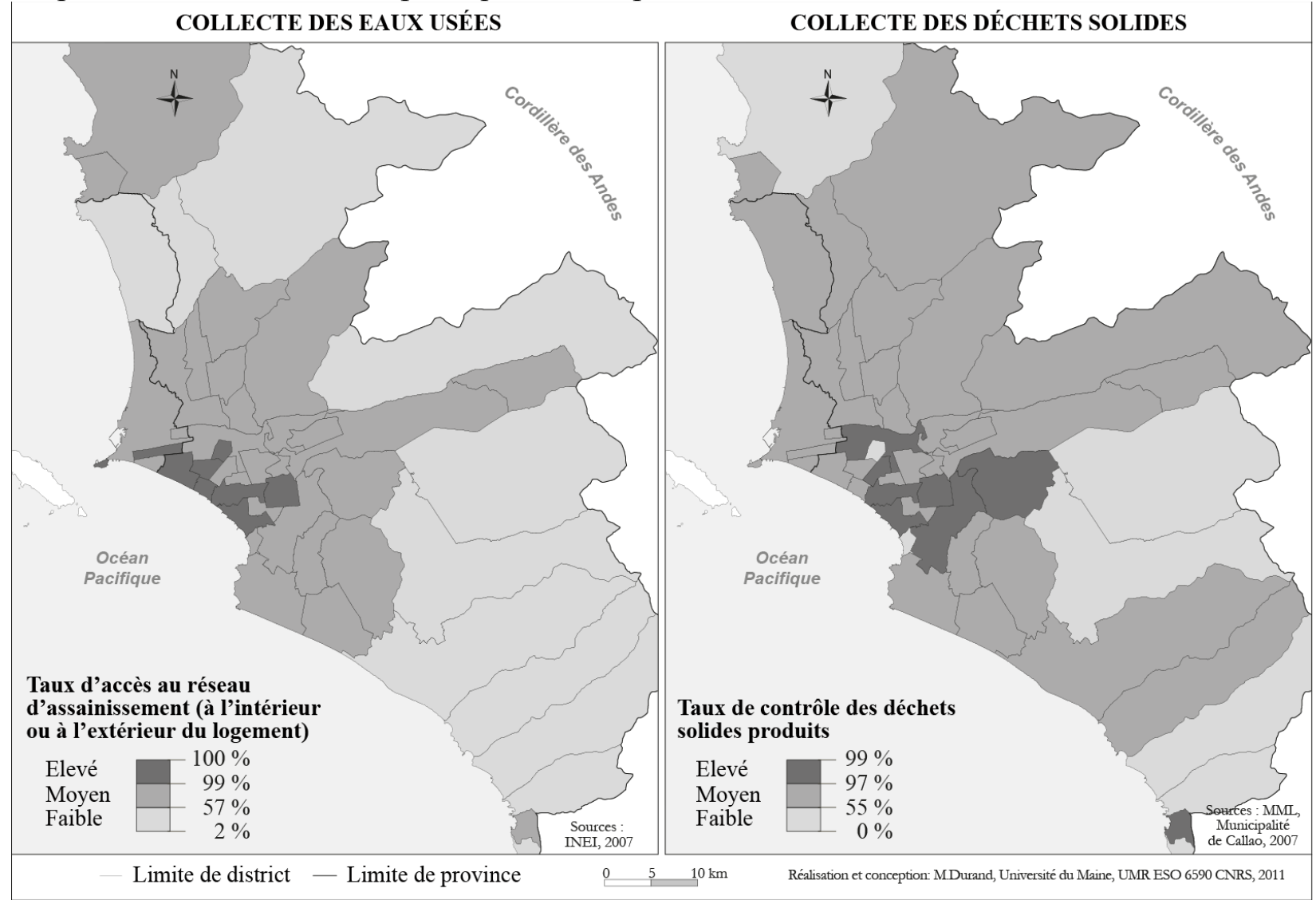

Figure $\mathrm{n}^{\circ}$ 4. Synthèse par habitant de l'accès au service public de gestion des déchets à Lima en 2007

Encore une fois, les observations réalisables pour les déchets solides et pour les eaux usées sont très similaires. On retrouve un taux d'accès au service public de collecte élevé dans la partie centrale de la ville, et faible dans les périphéries. On note cependant une différence importante. Le taux d'accès à l'assainissement est élevé à l'Ouest de la partie aisée de la ville, la plus anciennement urbanisée. C'est le fruit de l'installation ancienne des réseaux dans ce 
secteur. Au contraire le taux de contrôle des déchets solides est plus important dans la partie Est, correspondant aux quartiers plus récents et encore plus aisés. Ceci est le marqueur d'une capacité financière plus grande de ces municipalités, qui leur permet d'offrir un service efficace à leurs administrés. L'accès aux services publics de collecte reste ensuite à relativiser par rapport à l'effectivité du traitement et de l'élimination des déchets.

\section{II.3. La réception de déchets : une réception de nuisances}

La dernière étape permettant de mettre en lumière les inégalités environnementales et écologiques à Lima est celle de la réception des déchets. Les déchets collectés, selon les circuits formels ou informels, sont ensuite évacués vers des endroits spécifiques. Certains de ces espaces permettent d'éliminer les déchets, d'autres de les valoriser ou simplement de les évacuer. Les cartes de la figure $\mathrm{n}^{0} 5$ sont la synthèse d'un grand nombre de données spatialisées relatives à la réception de déchets.

Plusieurs degrés de risques ont été identifiés, correspondant à des différences de dangerosité des déchets (la nuisance) ou de vulnérabilité des populations. L'ensemble des données collectées sur le terrain, ainsi que la retranscription des discours des différents acteurs, ont permis d'établir cette classification, sans qu'elle corresponde à un indicateur qualitatif précis. L'appréciation de leur dangerosité peut alors être discutée. La quatrième classe d'objets de la carte («risque éliminé») localise les infrastructures permettant d'éliminer les déchets et donc de faire disparaitre la nuisance source de risques. Les éléments localisés sur la carte des eaux usées correspondent aux collecteurs d'eau usée de l'entreprise d'eau de la ville, aux zones agricoles directement ou indirectement irriguées avec des eaux usées, à la frange côtière particulièrement affectée par les eaux usées, ainsi qu'aux stations d'épurations (qui ne traitent que 14\% des eaux usées de la ville). La carte des déchets solides localise les décharges sauvages, les décharges contrôlées (Centres d'Enfouissement Techniques), ainsi que les zones de recyclage informel, ayant un caractère plus ou moins concentré (massif ou diffus). 


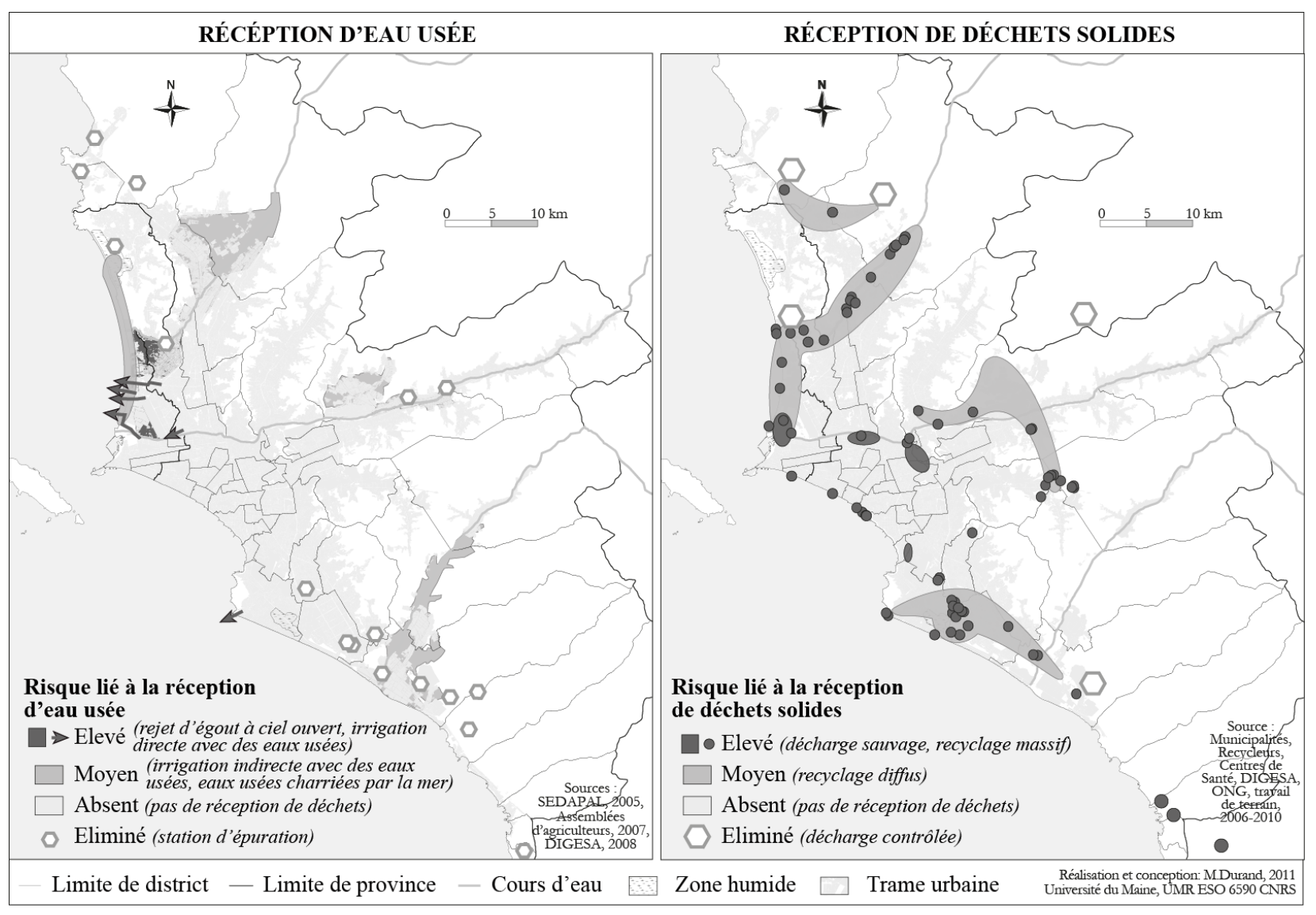

Figure $\mathrm{n}^{\circ} 5$. La réception de déchets comme indicateur de l'exposition aux risques

La localisation des territoires recevant les déchets est ainsi liée à l'emplacement des lieux de dépôt et de rejet officiels. Les zones agricoles utilisant les eaux usées se trouvent sur le passage des égouts de SEDAPAL. Les zones de recyclage et les décharges sauvages sont sur la route des décharges contrôlées. Ceci permet aux réseaux informels d'intercepter les déchets sur leur trajet vers leur destination finale officielle. Les territoires recevant les déchets sont presque tous situés dans les périphéries populaires de la ville. Seule une partie du recyclage informel se pratique à proximité immédiate du centre. Il s'agit alors de quartiers marginalisés socialement.

Les zones touchées par les déchets solides sont plus vastes que celle touchées par les eaux usées. Pourtant, $86 \%$ des déchets solides sont gérés dans les infrastructures municipales, contre seulement $14 \%$ des eaux usées qui sont traitées par les stations d'épuration. Si l'impact spatial des eaux usées est plus restreint, il est en réalité bien plus dangereux car la concentration d'eau usée est plus grande que celle des déchets solides. En raison de leur visibilité, les déchets solides dans l'espace public sont pourtant l'objet d'une plus grande attention. Par ailleurs, lorsque les déchets ne sont pas traités, la collecte permet simplement d'éloigner les nuisances et de déplacer le problème.

\section{Les inégalités environnementales et écologiques comme résultat de la gestion des déchets à Lima}

Les cartes de la section précédente ont repris un à un les indicateurs établissant le constat des inégalités environnementales et écologiques. Nous pouvons dès lors revenir aux définitions initiales de ces notions, construisant ainsi les lignes qui suivent en fonction de l'ordre des éléments de définition donnés dans la figure $\mathrm{n}^{\circ} 1$. Ceci permettra tout d'abord de 
faire le lien entre les données environnementales et les données sociales, ce qui a déjà été démontré par de nombreux travaux, mais qu'il est toujours important d'étayer davantage. L'analyse des données présentées précédemment permet ensuite, point important de la méthodologie développée ici, d'affirmer la distinction entre les inégalités environnementales et les inégalités écologiques, puis d'en comprendre les articulations. Cette distinction fait l'objet de nombreuses ambigüités dans les travaux scientifiques aussi bien qu'opérationnels, minimisant souvent l'amplification de leurs impacts croisés.

\section{III.1. Le constat des inégalités environnementales et écologiques}

La figure $\mathrm{n}^{\circ} 4$ montre que les individus sont inégaux face à la réception d'aménités environnementales, puisque tous n'ont pas accès au même service public de collecte des déchets solides et des eaux usées. Les districts de la ville aisée sont ceux qui ont pour l'essentiel accès aux réseaux de collecte. Les déchets sont évacués de l'espace public, limitant les nuisances pour les populations riveraines. Le différentiel d'exposition aux risques est alors le résultat de la réception d'une aménité, dont la mise en place est conditionnée par le degré de vulnérabilité sociale des populations ${ }^{6}$. Les quartiers où les populations sont les plus pauvres (cf. figure $\mathrm{n}^{\circ} 2$ et 6 ) n'ont pas la capacité de payer pour la mise en place d'un service public. La figure $n^{\circ} 5$ montre également que les quartiers recevant les déchets, c'est-à-dire recevant les nuisances et étant exposés aux risques sont également les quartiers les plus défavorisés socialement. Il s'agit des quartiers où la pauvreté et l'extrême pauvreté sont les plus importantes. Ce double constat caractérise les inégalités environnementales face à la gestion des déchets dans la ville de Lima.

Pour les inégalités écologiques, il existe encore une fois une très grande corrélation entre la production de déchets et le niveau socio-économique des populations. Les plus aisées sont celles qui ont des modes de consommation massifs induisant la production d'une plus grande quantité de déchets. L'empreinte écologique de ces populations est alors plus marquée puisqu'elles produisent plus de nuisances potentielles impactant l'ensemble de l'environnement urbain. Si l'élimination d'une partie de ces déchets permet d'éviter qu'ils ne soient source de nuisances pour les populations et pour l'environnement local, l'empreinte écologique due à leur production reste entière, puisqu'il n'y a que très peu de valorisation. Pour que l'annulation de l'empreinte écologique soit réelle, il faudrait opérer une valorisation des déchets, afin d'annuler l'effet des prélèvements de matières premières et de ressources naturelles. Or le recyclage des ordures et la réutilisation des eaux usées sont très faibles à Lima. Ils sont d'ailleurs limités aux pratiques des secteurs informels (recycleurs et agriculteurs), travaillant dans de très mauvaises conditions sanitaires et environnementales.

${ }^{6}$ L'articulation entre les déchets et les notions de transferts de risques et différentiels de vulnérabilité est détaillée dans un autre article (Durand, 2011) 


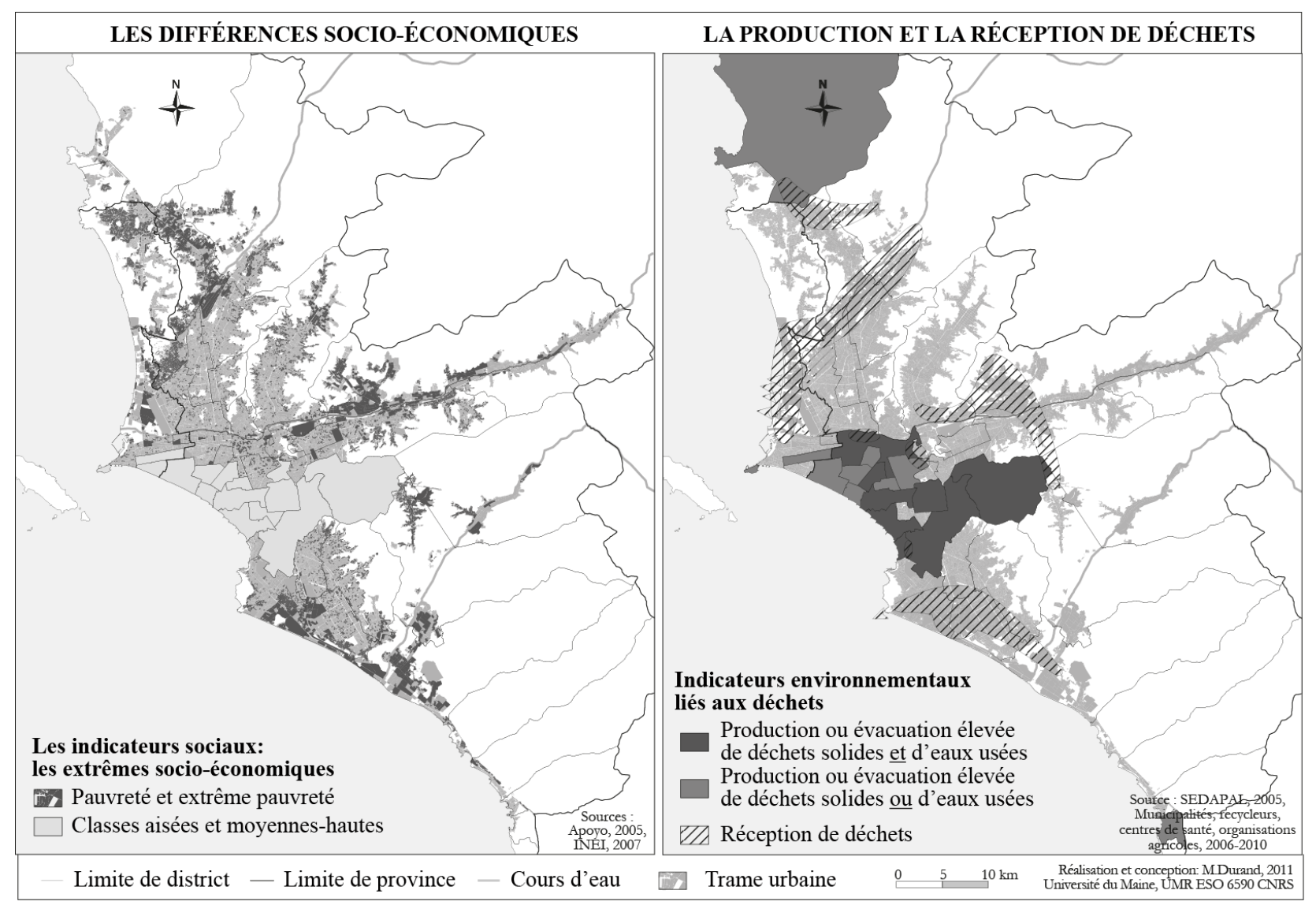

Figure $n^{\circ} 6$. Le constat des inégalités environnementales et écologiques à Lima

\section{III.2. Le cumul des inégalités dans la gestion des déchets}

La caractérisation de façon différenciée des inégalités environnementales et écologiques est importante, puisque qu'elle montre déjà de fortes discriminations. Elle permet ensuite d'avoir une approche plus distante et d'observer la façon dont elles s'articulent les unes avec les autres. La figure $\mathrm{n}^{\circ} 6$ superpose l'impact spatial des déchets solides et des déchets liquides sur la même carte. Elle permet de voir que les logiques spatiales relatives à la production et à la collecte des déchets sont pratiquement les mêmes ${ }^{7}$. Les populations produisant le plus de déchets sont généralement celles qui réussissent le mieux à les évacuer. On observe ici un premier cumul d'inégalités environnementales et écologiques faisant que les populations les plus aisées socialement peuvent produire de grandes quantités de déchets, sans être affectées par les nuisances associées. On peut considérer cette articulation comme un cumul des inégalités, puisque que les populations aisées ayant les moyens d'avoir un mode de vie avec une forte consommation matérielle, c'est-à-dire ayant une empreinte écologique plus grande et un impact important sur l'environnement (inégalités écologiques), sont également celles qui se défont des nuisances liées à cet impact (inégalités environnementales).

Au contraire, le second cumul concerne les populations les plus pauvres, produisant peu de déchets, mais ayant un accès défaillant à la collecte. L'impact de ces populations sur l'environnement est limité en quantité et en surface (puisque les déchets non collectés restent sur place). Ils n'ont pas la même «capacité à polluer» que les plus aisées et donc pas le même impact environnemental global (inégalité écologique). Par ailleurs, elles sont affectées par leur propre production de déchets, en plus d'être exposées à la réception des déchets

${ }^{7}$ Ce travail de synthèse n'est pas expliqué ici dans le détail. Il est le résultat du croisement de toutes les données utilisées dans la deuxième section de ce texte. 
provenant du reste de la ville, c'est-à-dire des populations les plus aisées (inégalités environnementales). Elles souffrent des nuisances liées aux déchets reçus (inégalités environnementales) sans pour autant les avoir produits (inégalités écologiques). Toutes les dimensions de ces inégalités viennent alors se cumuler pour fragiliser les mêmes populations.

La figure $\mathrm{n}^{\circ} 6$ montre que les districts où la population a une production élevée de déchets, ainsi que ceux où l'évacuation des déchets est élevée, concentrent la majeure partie des populations aisées de Lima. Ainsi concernant les eaux usées, ces districts représentent $98 \%$ des niveaux socio-économiques de catégorie $\mathrm{A}^{8}$ (populations aisées) et $58 \%$ des niveaux socio-économiques de catégorie $\mathrm{B}$ (classes moyennes-supérieures). Ces chiffres sont respectivement pour les déchets solides de $74 \%$ et $39 \%$. Au contraire, les quartiers recevant les eaux usées correspondent aux zones de pauvreté et d'extrême pauvreté selon les calculs d'estimation des dépenses effectués par l'IEP de Lima et retranscrits sur la figure $\mathrm{n}^{\circ} 6$.

Le constat de l'inégalité écologique est encore plus vrai pour les eaux usées. L'absence presque totale de traitement fait que toute eau usée produite, collectée et évacuée, aura un impact très fort sur les populations et sur les quartiers de réception. Le faible traitement des eaux usées et la petite taille des espaces où elles sont accumulées, notamment la baie de Callao, provoquent une très forte concentration de la nuisance et des risques d'autant plus élevés. Au contraire, la dispersion de la réception des déchets solides permet d'en diluer les impacts et d'en diminuer la dangerosité. La situation ici décrite est postérieure à l'an 2000, puisqu'avant cette date les déchets solides municipaux étaient également peu traités. La prise de conscience de l'importance du traitement des déchets est donc bien réelle à Lima. Les autorités tentent aujourd'hui d'offrir un traitement plus complet des déchets et de ne plus simplement les évacuer de l'espace public. Malgré tout, le coût très élevé des stations d'épuration ne permet pas leur construction à court terme. La rigidité des infrastructures de gestion des eaux usées (réseaux, stations d'épuration) est également un frein à leur mise en œuvre rapide. La gestion des déchets solides peut se contenter d'infrastructures moins lourdes, plus éparses et faire intervenir plusieurs types d'acteurs. La dispersion de la gestion des déchets solides est favorisée par un maillage de recycleurs intervenant dans l'ensemble de la ville. La visibilité de leur activité est grande, contrairement aux eaux usées évacuées dans les souterrains, et ce maillage permet de tirer un profit économique et environnemental important en valorisant les déchets solides.

\section{III.3. Et si ces inégalités permettaient à la ville de fonctionner...}

La gestion des déchets est ainsi très souvent caractérisée par un cumul des inégalités environnementales et écologiques dans les villes en développement. Or ces inégalités permettent pourtant au système de gestion des déchets de fonctionner. C'est en effet grâce aux populations souffrant des inégalités, c'est-à-dire recevant les déchets, que le système est opérationnel. Sans l'appui des recycleurs informels, une part plus importante de déchets ne serait pas collectée, notamment dans les quartiers les plus pauvres, là où le manque d'infrastructures ne permet pas aux municipalités d'intervenir. Le recyclage des déchets, point d'orgue des politiques de développement durable, ne serait pas non plus réalisé sans l'intervention des recycleurs. Sans le penser comme tel, ces populations sacrifient leur propre santé, permettant ainsi indirectement au système urbain. La vulnérabilité de certains habitants de la ville est source d'inégalités mais permet aussi de réduire la vulnérabilité de la ville en jouant sur autre volet de son métabolisme urbain (Coutard \& Lévy, 2010). La situation est

${ }^{8}$ Ces catégories sont celles qui ont permis de localiser les populations les plus aisées de la ville sur les figures $\mathrm{n}^{\circ} 2$ et 6 . Ces données sont produites par 1'Institut Apoyo (2005). La catégorie A correspond aux populations les plus riches et la E aux plus pauvres. 
identique pour les eaux usées, puisque les agriculteurs les réutilisant sont les seuls dont les pratiques permettent une certaine valorisation.

La nécessité de reconnaitre les inégalités risque donc de ne pas être suffisante pour pouvoir les réduire. Le système actuel, basé sur une articulation entre les systèmes formels et informels (caractéristique du système composite, Jaglin, 2007) ne fonctionnerait en effet pas sans les inégalités structurelles entre les populations urbaines. Il faut agir plus en profondeur pour réformer le système de gestion des déchets et pour aller vers une intégration progressive de tous les travailleurs des déchets, vers le système formel. La difficulté de pouvoir mesurer et cartographier les inégalités environnementales et écologiques, ne doit pas minimiser la compréhension de leur construction, lorsque celle-ci est inhérente au mode de fonctionnement de l'espace urbain. Cette compréhension n'est pas détaillé ici, puisque l'objectif de ce texte est d'esquisser une méthodologie de mesure des inégalités, pas toujours évidente à mettre en œuvre.

Un modèle de développement durable doit ainsi chercher à limiter les inégalités relatives à l'environnement, les articuler avec les inégalités sociales, sans pour autant détruire brutalement le système qui permet à la ville de fonctionner.

\section{Bibliographie}

- BARRAGÁN ARQUE Paulino, 2005, Magnitud de la economía informal en el Perú y el mundo, Revista gestión en el tercer milenio vol.7 nº14, Lima: UNMSM, p.43-51.

- BELLAN Gérard, BELLAN-SANTINI Denise et DAUVIN Jean-Claude, 2007, $A$ propos de quelques utilisations des termes "Inégalités écologiques" : simples impropriétés de langage ou accaparement abusif?, revue Développement durable et territoire, Dossier 9: «Inégalités écologiques, inégalités sociales », 15p.

- CALDERON Julio, 2009, La producción de la ciudad formal e informal, dans Foro urbano, "Los nuevos rostros de la ciudad de Lima", Lima: Colegio de sociólogos del Perú, p.17-30.

- COUTARD Olivier, LEVY Jean-Pierre (dir.), 2010, Ecologies urbaines, Paris : Economica-Anthropos, $371 \mathrm{p}$.

- DUBOIS Jean-Luc, 2001, Pauvreté et inégalités : situation et politiques de réduction, en LERY A., VIMARD P., "Population et développement : les principaux enjeux cinq ans après la conférence du Caire », Les documents et manuels du CEPED n ${ }^{\circ} 12$, Paris : CEPED, p.123-135

- DUCHEMIN Jean-Paul., 2004, Déchets et assainissement: quel rôle pour la commune?, Programme «Gestion durable des déchets et de l'assainissement urbains », Paris : PSEAU, PDM, 24p.

- DURAND Mathieu, 2010, La gestion des déchets et les inégalités environnementales et écologiques à Lima, entre vulnérabilité et durabilité, Rennes: Université de Rennes 2 , Thèse de doctorat, $466 \mathrm{p}$.

- DURAND Mathieu, 2011, La gestion des déchets et les inégalités environnementales et écologiques à Lima, entre vulnérabilité et durabilité, Rennes : ESO Travaux et documents $\mathrm{n}^{\circ} 32$, A paraître.

- EMELIANOFF Cyria, 2006, Connaître ou reconnaître les inégalités environnementales?, Rennes : ESO Travaux et documents $\mathrm{n}^{\circ} 25, \mathrm{p} .35-43$.

- EMELIANOFF Cyria, 2010: Connaître ou reconnaître les inégalités environnementales?, dans DJELLOULI Yamna, EMELIANOFF Cyria, BENNASR Ali, CHEVALIER Jacques, "L'étalement urbain, un processus incontrolable?», Rennes : PUR, p.221-234. 
- GOUESET Vincent, 2006, Des populations marginalisées? Pauvreté et inégalités socio-raciales, dans DUREAU Françoise, GOUESET Vincent, MESCLIER Evelyne, " Géographie de l'Amérique Latine », Rennes : PUR, p.111-118

- JAGLIN Sylvy, 2007, Décentralisation et gouvernance de la diversité, les services urbains en Afrique anglophone, dans FOUCHARD Laurent, "Gouverner les villes d'Afrique : Etat, gouvernement local et acteurs privés », Paris : Karthala, p.21-34

- LAIGLE Lydia, 2004, Les enjeux sociaux et environnementaux du développement urbain : la question des inégalités écologiques, Paris : PUCA-MELT, 112p.

- LE BRIS Émile, 2002, Inscrire la question de l'assainissement et des déchets dans une problématique urbaine, Programme «Gestion durable des déchets et de l'assainissement urbains », Paris : PSEAU, PDM, 17p.

- RIOFRIO Gustavo, 2009, Imágenes y perspectivas del crecimiento urbano de Lima, en Foro Urbano, "Los nuevos rostros de Lima", Lima: Colegio de sociólogos del Perú, p.31-50.

- SEDAPAL, 2005, Plan Maestro Optimizado, Lima: SEPAPAL, Vol.1.1 125p., Vol.1.2. 234p., Vol.1.3. 71p., Vol.2.109p., Vol.3.1.69p., Vol.3.2. 272p., Vol.3.3. 137p., Vol. 3.4. 1345p., Vol.3.5. 508p., Vol.4. 94p.

- THEYS Jacques, 2002, L'approche territoriale du "développement durable », condition d'une prise en compte de sa dimension sociale, revue en ligne Développement durable et territoires, dossier $\mathrm{n}^{\circ} 1$ : «Approches territoriales $\mathrm{du}$ développement durable $»$, $13 \mathrm{p}$. http://developpementdurable.revues.org/index1475.html

- WACKERNAGEL Mathis, REES William, 1999, Notre empreinte écologique, Montréal : Editions Ecosociété, 207p. 